\title{
Predicting Innovation Acceptance by Simulation in Virtual Environments (Theoretical Foundations)
}

\author{
Noel León ${ }^{1}$, Roberto Duran ${ }^{1}$, Humberto Aguayo ${ }^{1}$, and Myrna Flores ${ }^{2}$ \\ ${ }^{1}$ Center for Innovation in Design \& Technology (CIDT), ITESM, \\ Garza Sada 2501, 66849, Monterrey, Mexico \\ ${ }^{2}$ CEMEX Global Center for Technology and Innovation, CEMEX Research Group AG, \\ Brügg b. Biel, Switzerland, Processes and IT, Research and Networking \\ \{Noel Leon, Roberto Duran Humberto Aguayo, Myrna Flores, \\ noel. leon\} @itesm.mx
}

\begin{abstract}
This paper extends the current development of a methodology for Computer Aided Innovation. It begins with a presentation of concepts related to the perceived capabilities of virtual environments in the Innovation Cycle. The main premise establishes that it is possible to predict the acceptance of a new product in a specific market, by releasing an early prototype in a virtual scenario to quantify its general reception and to receive early feedback from potential customers. The paper continues to focus this research on a synergistic extension of techniques that have their origins in optimization and innovation disciplines. TRIZ (Theory of Inventive Problem Solving), extends the generation of variants with Evolutionary Algorithms (EA) and finally to present the designer and the intended customer, creative and innovative alternatives. All of this developed on a virtual software interface (Virtual World). The work continues with a general description of the project as a step forward to improve the overall strategy.
\end{abstract}

Keywords: CAI, GA, TRIZ, Theory of Inventive Problem Solving, Evolutionary Algorithms, Virtual World.

\section{Introduction}

Given the importance of a reliable and successful introduction of new products into the market and the need to assure the return of investment of R\&D projects, industry and the related development support partners, such as Universities and consulting research firms, are looking for innovative ways to increase the success rate of the ideation, development, launching and commercialization of new products worldwide. Virtualization, as an alternative way enable people to communicate and interact with the developers, therefore nowadays has an increasing impact on the life of more individuals. At the same time virtualization provides benefits to innovative companies, as these new tools allow their customers to provide their preferences in a more effective and entertaining way. Additionally, this brings another challenge, as in the innovation process, given the wide range of alternatives, it is very important to filter the most promising solutions to impact the adequate market. Virtualization is especially 
relevant to reduce the variation from the conceptual design of the product targeting what customer value the most. Therefore, the authors are conducting a series of experiments related to this business opportunity, facing different perspectives and tools with a case study for the construction industry.

The main goals of the presented research are, to construct a strategy for the development of solutions based on a Computer Aided Innovation (CAI) framework and to build the foundations for a research topic that utilizes different tools as an interfaces between the designers and their customers. Being EA the main optimization and innovation mechanism, through shape construction and editing inside a virtual environment, the developer could test and prove the acceptance of different alternatives, and the computers could reach the boundaries of creativity. This paper describes the conceptual architecture of our proposal rather than the technical implementation details.

\section{Background}

Different disciplines have been converging to form the groundings of this research topic on CAI. Evolutionary design [1] is a process that can generate designs from changing forms and shapes based mainly in Genetic Algorithms (GA's), a particular case of EA. Concepts evolve gradually from a population that is submitted to operators that mimic the biological evolution: selection, mutation and crossover. But, although GA's show impressive results compared to other search engines, it is mainly used for optimization, even when it is perceived by many as a potential tool for creativity enhancement in both conceptual [2] and practical contexts [3]. Darwin's idea of 'survival of the fittest' means that the most adaptive organisms in a population will survive through the 'natural selection' process. This gradual process takes place in a relatively stable environment, working slowly over long periods of time necessary for the distinctive characteristics of species to show their superiority in the 'survival contest'. But this gradual process can be accelerated, working in a virtual environment over shortened periods of time, being the 'fitness' the acceptance in such virtual worlds.

The new requirements of products are in a constant change, and companies are trying to catch the market needs in order to update their products before their competitors by finding fast decision variables. EA represents a search of solutions in an evolving sequence that can be used to find and answer these variables in an accelerated environment. Evolutionary Design takes the discipline of CAD and extends the design capabilities for what they have been used until now. Moreover, as the processing capabilities of computers continue increasing, the resolution of more complex Virtual models is improved. 'Virtual Selection' (VS), thus, is a gradual process that selects the 'fittest' organisms inside a computer framework to derive on CAI. The process of VS eliminates poorly adapted variants in a compulsory manner, normally by engineering simulation such as Computer Fluid Dynamics, Structural Finite Element, etc. But also compulsory consumer attitudes of virtual costumers are possible to be investigated, since individuals who are 'unfit to the world' are supposed to have no way of escaping the consequences of non acceptance. A parallel search criteria can be developed taking in consideration both engineering and social/market virtualizations.

Computational social simulations have been developed to address a number of questions about the multiple links between creativity and innovation [4]. Virtual Reality has different trends for the search of a simulation with such social implications, 
represented in commercial on-line software. Virtual reality can serve to new product design, helping as a tool for engineering in manufacturing processes, new product prototype and simulation. Among other examples, CAD, Finite Element Analysis, and Computer Aided Manufacturing are used with an increase in virtual representation such as Factory and Manufacturing process simulation. These tools can be derived on an extended prototyping method, achieved by representing products for early introduction to the market.

The variation of products intended for this experiment is controlled by Genetic Algorithms that represent the geometrical alternatives. This eliminates the parametric restrictions on physical prototypes and allows many concept variations of forms and possibly of functions, when professionals are involved in the evaluation pool. Also, the experiment could produce automatic variations in 3D shapes and have concept variations evaluated by software. In other words, GA's controlling virtual shapes are an adequate interface to apply innovation operators from TRIZ (or other innovation methodology) to the development of a product in a virtual environment. Other authors have presented novel visual evolutionary computing environments to provide support for creative design [5]. 2D and 3D images were generated by combination of evolutionary computing technology and visualization technology in computer environments.

In a competitive market place, the strategy for developing products has to be transformed from a product based to a market focused model, since the Kano model [6] explains that excitement characteristics of a product are satisfied needs that make the difference in a mature market (see fig. 1). Facing the intense changes in the world, a well-designed product should not only satisfy consumers' basic material requirements but also satisfy their psychological excitement expectations. Design must comply with performance, quality and visual satisfaction. This requires that designers and engineers are able to design with knowledge and tools for preventing the disappointment of excitement expectations of potential customers. This paper presents a new way of using evolutionary computing technology and visual virtualization technology to support creative design.

Our goal is to give more opportunities to designers to be creative by unleashing the creative potential with computational environments. The approach to support creative design is going to be by the development of computational tools that can generate sketches and images that inspire the designers in the creative design process, and also the analysis of results in a virtual environment. Forms of virtual representations produced as prototypes are also a type of evaluation resources that designers could test. Designers then could apply the results to their design and produce an improved design. In product design, visual representations, in the form of sketches or bodies, is a key activity in the process of originating new product ideas.

\section{Virtual Scenario Planning for Innovation}

In order to achieve their business objectives, companies typically conduct an innovation process using the following tools: interviewing customers, performing Quality Function Deployment (QFD) workshops, brainstorming, formulating new product concepts, creating prototypes, testing them in the market and refining concepts with customer feedback. The accepted traditional methods for carrying out innovation processes offer no guarantee of success. It is well understood, for example, that direct 


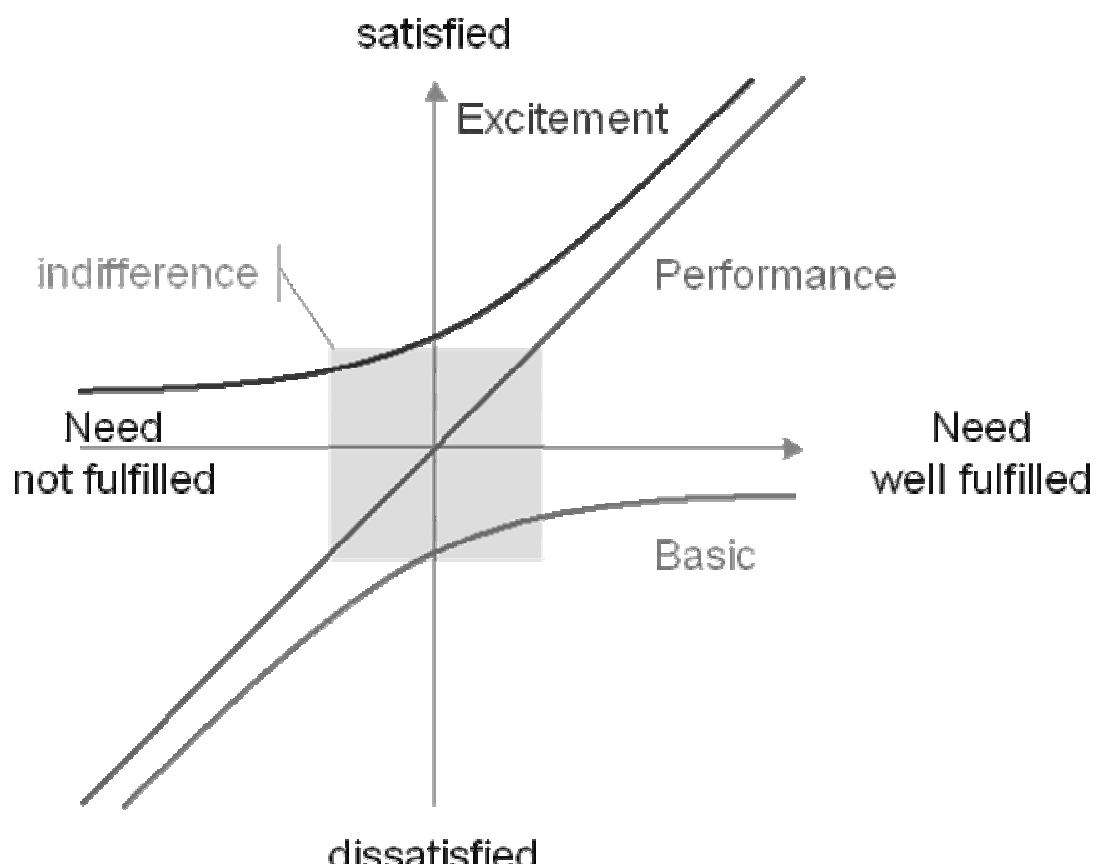

Fig. 1. Kano model for representation of basic vs. excitement needs

use of "voice of the customer" for innovation too often leads to customer disappointment or market failures [7]. Despite the best efforts to reduce the risk and avoid failures, about $70 \%$ of all industry innovation initiatives are abandoned or fail. Of those innovations that do succeed, over $80 \%$ offer only incremental improvements compared to the products on the market. That explains the high level of interest and expectation on the computer-aided innovation and invention tools, including TRIZ methodology and Virtual Scenarios. Others have defined the term Unexpected discoveries as key factors to generate new ideas [8].Our approach under study will use Virtual Scenarios to help anticipate and calculate different market opportunities for innovation concepts already in the very early stages of the innovation process - in the phase where the innovation strategy and tasks are formulated. It is intended to reduce innovation costs and risk of poor investments. In fact, in their last report about the Virtual Worlds market, Garter [9] suggests to all enterprises to investigate applicable uses of this technology to increase their competitiveness as they enable people to interact for innovation.

\section{Application Example under Development}

The present case study consists on the development of an experiment to test the acceptance of a product from the construction industry, means the introduction to the virtual environment. The product to be modeled and tested by end-users thanks to virtual world technology is a "green house" model, where the acceptance of the 
proposed innovation is going to be monitored in the virtual environment. The "green house" concept is an emerging because it focuses to provide new solutions to world wide shortages of non renewable resources, such as water and reduce increasing costs of energy and waste management. Several factors are impeding the fast introduction of the "green house" in the market, for instance: Current technology is relatively expensive for mass implementation (specially in developing economies), designs and product performance are still in an infancy stage and many customers lack awareness of the benefits and savings they can obtain if they adopt these new technologies for sustainable housing. Therefore, this kind of product is very suitable to be investigated in a virtual environment, where different scenarios can be proposed and tested by final users who can provide feedback of their preferences and at the same time "learn" what a "green house" is without the need to fully construct it for demonstration purposes. In the long term, this will also increase their satisfaction and excitement levels as proposed by the Kano Model mentioned previously in this paper.

From a standard shape consisting of lines and arcs to the geometry of the platform which is going to be introduced and finally a set for experimentation was defined. The modifications of the control points produce changes in the geometry of the product that result in an acceptance response. The fitness function selected as objective is the acceptance differences between the acceptance response of the individual configuration and the target specified as a design requirement. The algorithm will then try to optimize the absolute deviations from the differences.

The parametric virtualization software that is intended to be used during and further the experimentation stage, could be automatically manipulated by Genetic Algorithms means an interface programmed in script language. One of the characteristics of the virtual interface needs to be that the script is continually running with the products model loaded in the server. This means that every time an individual is generated, the geometry adapts to the parameters automatically. In other words, the characteristics are going to be introduced into the software in a manner that automatically provide the values for the parameters. These values will automatically be updated every time the geometry is modified, when an interface to link the CAD model to the GA is implemented.

\subsection{Specific Requirements to Be Satisfied}

- Measuring and control the acceptance of the different variants of products by developing different scenarios and interacting with customers in the virtual world to obtain their preferences.

- Developing the programming interface that allows the generation of the different alternatives: It will be used a Script program for the manipulation of the model modifications during the Virtual simulation. Genetic Algorithms will be used for optimization and search of alternatives.

- Finding the innovation principles that complement the genetic operators (mutation, crossover, etc) in order to generate a list of "virtual-real" innovation correlations.

- Obtaining new design concepts for the construction sector that will help designers, architects and civil engineers to build novel sustainable building in terms of functionality, space utilization, energy efficiency, waste management,etc.

Another interesting requirement could be the restriction of genetic representation based on the manufacturing capabilities of the user, or even market criteria, but at this stage those are not considered "mandatory" requirements. 


\subsection{Implementation (Technical Solution for Development)}

Recently, computers have taken over much of the simulation role. The simulation technique works as follows: A model of some aspect of the real world is transferred to the computer. Through the simulation technique, final customers can test the usability of new product and simulate its performance output and potential operational failures that it may have. In each case, the whole process takes place inside the computer at a small fraction of the time it would take in the real world. Of course, it is necessary to be careful because the models are only approximations. No simulation can predict exactly what will happen in reality, but a good simulation is by far preferable to blind trial and error. Visionaries that can simulate the future are ahead from whom can only learn on the basis of trial and error. The problem with trial and error is that it takes time and energy, and the error is often fatal, as it might consume the development budget of the innovation project. Simulation is both safer, faster and cheaper for testing the acceptance and usability of the product under study.

An acceptance criterion for the solutions is going to be implemented in the solution. Next is described the conceptual architecture of our framework. Fig. 2 shows the intended implementation.

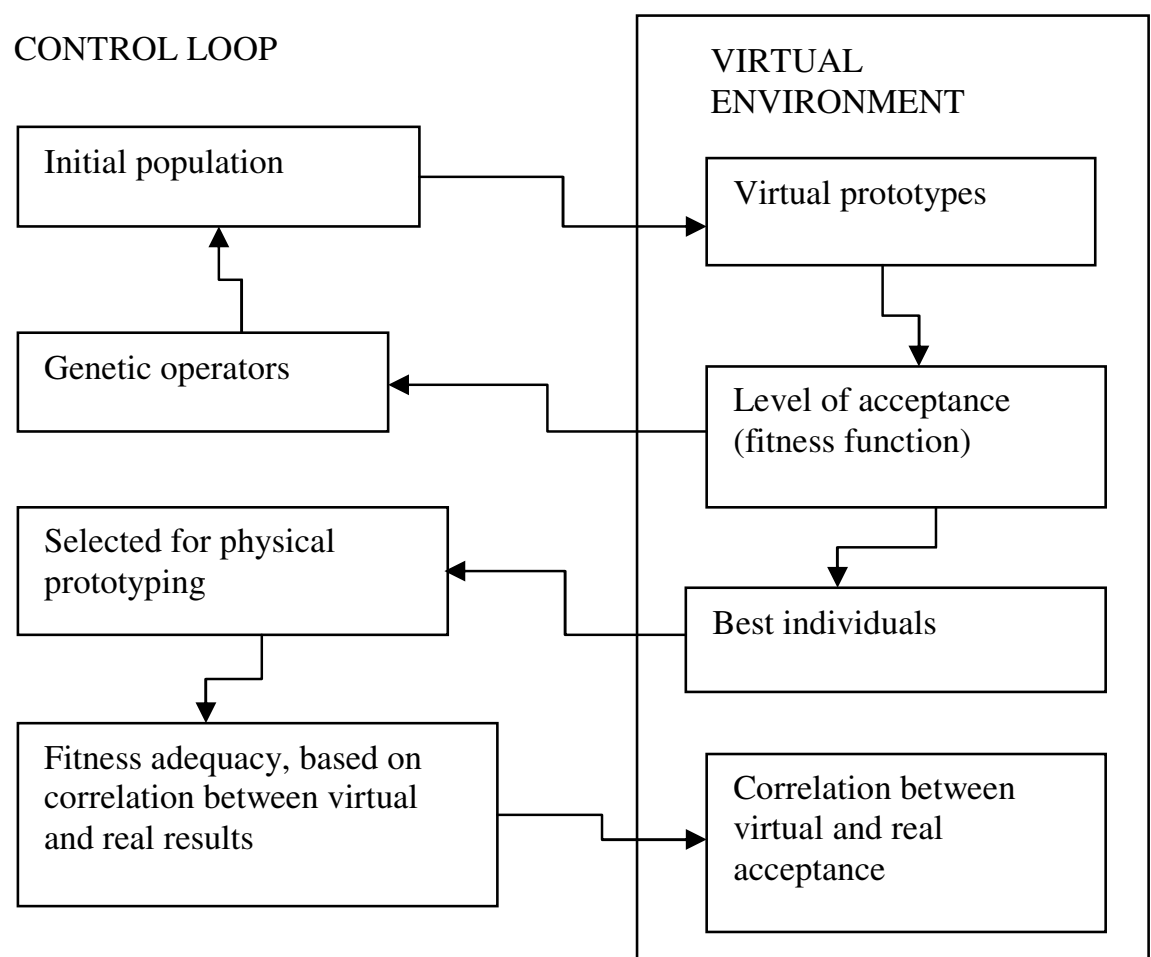

Fig. 2. Scheme of implementation for the virtual model 
However, the simulation is not the only instrument which can solve the development problems; it is possible to use our minds by what Dawkins [10] called the extended phenotype. The phenotypic effects of a gene are usually seen by their effect on the body in which it resides. This is the conventional definition, but the phenotypic effects of a gene can have any impact on the outside world and possible in a virtual world. The effect of a gene, in fact, is not confined to organisms where the gene resides, it may influence the body of an external individual. What might it mean in practice to speak of a gene that phenotypically affects the world outside the body in which is sitting? It means the possibility of the particular gene to influence the mind of the designer in order to stimulate him/her to generate disruptive ideas. This has importance when a person who is working with the process or product, can intervene at any moment and introduce new "ideas" not directly derived from the evolution but inspired at the moment of observation. The effect of having some intermediate results can give the designer disruptive configurations of how to improve the system, and thus affect a generation not only as an optimization but as a conceptual leap (quantitative turns into qualitative).

\subsection{New Tools for Modeling and Developing in Virtual Scenarios}

Next are some pictures key characteristics provided by virtual modeling tools to provide an impression of the digital scenarios to be developed. Their characteristics are as follows:

1. Flexible and customizable interface / non-intuitive

2. Implementation for the rapid creation of organic models and skeletons (Rigs).

3. Easy to work with polygonal models (possibility to implement genetic algorithms in the model)

4. Easy to edit finished models (tweaking)

5. Focused on customers' details, perfect for organic surfaces or creating hyperrealistic textures.

Virtual modeling tools have extensive documentation, and some have support from virtual worlds. Some software is much like a 3D modeling program and are classified as "sculpture" (similar to working with clay), other software is programmable and the models can be manipulated by a programming language (which will be used to manipulate the individuals of each generation during an evolution). However, this characteristic list is not for a CAD format and it would be a great opportunity to use new tools for the case studies planned to be realized.

\section{Conclusions}

The use and combination of the tools on virtualization and innovation capabilities are intended to be a mean to "predict" the acceptance of products by variation of shapes, forms and topologies derived from the simulated evolution. EA are viewed as the mechanism to embed the innovative principles in a virtual interface. For instance, extend the functionality of a product into a growing market that is faster and cheaper to experiment with, and then evaluate its possibility in the real world. Even if the previously mentioned do not work as it is intended, it is still possible to share opinions among collaborators or receive feedback about a product in a more dynamic and semi-automated way. 
The best innovation and business success opportunities possess benefits that are important to customers but are not satisfied by existing technical solutions, products or services. Selecting benefits with the highest market and innovation potential from a long list of customer benefits is vital, since chasing after less promising ones consumes resources. The developed computer-aided virtual tool for innovation concept acceptance makes it possible to discover the most promising areas for further design work, bridging computer aided innovation with the computer-aided design. This is a step towards the generation of a Computer Aided Inventing environment that deepens in the evolution of technical systems as an analogy with the biological evolution. The basics of a new concept of virtual design software and computer aided inventing (CAI), are presented and its impact on global design performance and limits exploration is analyzed. Especially, the ability to introduce automatic changes in shapes and topologies in a 3D-Virtual environment are linked to the concept of CAI allowing exploring the space of possible solutions to a design problem.

\section{References}

1. Bentley, P.: Evolutionary Design by Computers. Morgan Kaufmann, USA (1999)

2. Gero, J.: Creativity, emergence and evolution in design 9, 435-448 (1992)

3. Koza, J.R., Keane, M.A., Streeter, M.J., et al.: Invention and creativity in automated design by means of genetic programming. Artif. Intell. Eng. Des. Anal. Manuf. 18, 245-269 (2004)

4. Sosa, R., Gero, J.: Computational Explorations of Compatibility and Innovation. Trends in Computer Aided Innovation (2007)

5. Liu, H., Tang, M., Frazer, J.H.: Supporting creative design in a visual evolutionary computing environment. Advances in Engineering Software 35, 261-271 (2004)

6. Matzler, K., Hinterhuber, H.H.: How to make product development projects more successful by integrating Kano's model of customer satisfaction into quality function deployment. Technovation 18, 25-38 (1998)

7. livotov, P., Murnikov D.: Computer-aided approach for scenario planning of innovation strategies and its application for innovative concept development in software engineering, Germany (2005)

8. Tan, R.: UXDs-Driven Contradiction Solving For Conceptual Design Using CAIs. Trends in Computer Aided Innovation (2007)

9. Prentice, S., Rozwell, C., Harris, M., et al.: Virtual Worlds: What to Expect in 2009 (Feburary 2009)

10. Dawkins, R.: The selfish gene. Oxford University Press, Oxford (1989) 